\title{
Robots for Field Operations with Comprehensive Multilayer Control
}

\author{
Jæger, Claes Lund Dühring; Griepentrog, H. W.; Paraforos, D. S.
}

Published in:

$\mathrm{KI}$ - Künstliche Intelligenz

Link to article, DOI:

10.1007/s13218-013-0266-z

Publication date:

2013

Link back to DTU Orbit

Citation (APA):

Jæger, C. L. D., Griepentrog, H. W., \& Paraforos, D. S. (2013). Robots for Field Operations with Comprehensive Multilayer Control. KI - Künstliche Intelligenz, 27(4), 325-333 . https://doi.org/10.1007/s13218-013-0266-z

\section{General rights}

Copyright and moral rights for the publications made accessible in the public portal are retained by the authors and/or other copyright owners and it is a condition of accessing publications that users recognise and abide by the legal requirements associated with these rights.

- Users may download and print one copy of any publication from the public portal for the purpose of private study or research.

- You may not further distribute the material or use it for any profit-making activity or commercial gain

- You may freely distribute the URL identifying the publication in the public portal

If you believe that this document breaches copyright please contact us providing details, and we will remove access to the work immediately and investigate your claim 


\title{
Robots for Field Operations with Comprehensive Multilayer Control
}

\author{
H.W. Griepentrog • C.L. Dühring Jaeger • \\ D.S. Paraforos
}

Received: 21 March 2013 / Accepted: 11 June 2013

(C) Springer-Verlag Berlin Heidelberg 2013

\begin{abstract}
Today research within agricultural technology focuses beside productivity and operation costs mainly on increasing the resource efficiency of crop production. Autonomous machines have the potential to significantly contribute to this by utilizing more multi-factorial real-time sensing and embedding artificial intelligence. A multilayer controller has successfully been implemented on two outdoor machines with various implements to conduct several agricultural applications in autonomous mode. Future work has to be conducted to achieve a more integrated and flexible implement control.
\end{abstract}

Keywords Autonomous field operations · Controller software framework - Autonomous weed control

\section{Introduction}

Today research within agricultural technology focuses not only on productivity and operation costs but increasingly on resource efficiency of crop production. Efficiency in production can be optimized by improving machine functions, behaviors and coordination as well as by optimizing crop growing conditions via improving the production process in general. Small and smart autonomous machines have the potential to significantly contribute to both by utilizing more multi-factorial real-time sensing and embedding artificial intelligence by using mathematical modeling of technical and biological parameter interactions.

H.W. Griepentrog $(\bowtie)$ · C.L. Dühring Jaeger · D.S. Paraforos Institute of Agricultural Engineering, University of Hohenheim, Garbenstr. 9, 70599 Stuttgart, Germany

e-mail: hw.griepentrog@uni-hohenheim.de
Despite the progress of incorporating smartness of control into production processes there are still challenges remaining:

- Sensing and information retrieval to acquire knowledge about more relevant process parameters

- Stored information on systems in individual as well as distributed data bases do not show compatible data structures

- Integration of mission planners into Farm Management Information System (FMIS)

- Fusion of information by using models and algorithms for Decision Support System (DSS)

- Methods from artificial intelligence (AI) for robot control not yet used as integrative discipline

Small robots fit better to small scale landscape structures than conventional big machinery, and hence ponds, trees, ditches etc. do not have to be removed which is still the case in some regions. Therefore, this would contribute to protect the environment and keep high levels of biodiversity of landscapes. Even a reintroduction of elements could be considered, because they would not have a negative effect on machine capacities. Today in general no field structures should be modified to meet machine requirements as done during green revolution in the 70es. The structures should rather be kept by maintaining complex and divers landscapes, but new smart and small robotic machinery would have no negative influence on machine productivity and the economic viability of the production process.

During the past decade research prototypes for conducting autonomous operations have been developed. The aim of the paper is to give examples of existing complex autonomous machinery being able to execute agricultural field operations. The paper is structured into introduction to the problem, state-of-the-art of autonomous machinery fol- 
lowed by descriptions of hard- and software of two different autonomous machines and their implements.

\section{Autonomous Platforms}

Today robots and autonomous machines have been investigated and used for several agricultural applications $[2,3,14,15,23]$. Furthermore, a yearly student competition with small robots is conducted, where tasks are field operations with an agricultural background [28].

Beside robots having combustion engines more and more electric powered robots for outdoor performance get popular due to several advantages. The machine control is easier in terms of sensor integration, active navigation and task application compared with traditional machine types. Furthermore, low machine weights and the use of renewable energy to provide the necessary energy contribute to soil protection and low emission performance. Currently electric powered robots are costly while most of the commercial available robots are not modular in design. Modular designed robots can be adapted to and used for different tasks and in different environments. Modularity also contributes to acceptable prices $[13,17]$.

Autonomous machines for field operations can be developed based on two different approaches: The deterministic and reactive behavior [3]. For the deterministic type, the navigation as well as the application is planned by the operator in advance before the autonomous execution. This requires time consuming thorough considerations and planning and for the execution often only a GNSS based system is needed. For allowing reactive behavior in navigation and application real-time sensing and data processing is needed. For this approach appropriate sensors, suitable analysis routines as well as fast computational processing are needed to realize on-the-go execution. In order to improve the tracking control performance, various soft computing methods as neural networks [20], fuzzy logic [19], and evolutionary algorithms [16] have also been applied to reactive control systems.

Although reactive navigation architecture establishes successful schemes for robot control, there are still some drawbacks regarding complex environments, because overall goal-oriented targets are not taken into consideration as in deterministic control. Hybrid control adopts the best properties of the reactive and deterministic control architecture. It combines the real-time response of reactive control with the rationality and optimality of deterministic approach [29].

\subsection{The Autonomous Mechanization System (AMS)}

For a conventional $20 \mathrm{~kW}$ tractor (Hakotrac 3000) the steering wheel, the driver seat and the cabin were removed. The machine was retrofitted with a controller system consisting of a navigation computer and an electronic control unit (ECU) to act as an interface between the computer and the functions on the tractor. Sensors were added to provide positional information (GNSS) as well as feedback for close-loop-control of parameters (steering angles, shaft speed, linkage height etc.). Two electro-hydraulic valves were added to actuate the front steering wheels and electric linear motors control engine rpm (Diesel injection) and forward speed by the continuously variable transmission (CVT).

Implements can be added to the linkages in the rear, front and machine center. The tractor navigation controller was designed to follow a predetermined route plan accurately and repeatable across a field with planned action points for implement control [4]. Several implements that allow different field operations have been developed for the machine, for example precision seeding and weeding tools (Fig. 1).

\subsection{The Armadillo Scout}

A small robot (Fig. 2) is based on the Armadillo Scout machine which was developed by both the University of Southern Denmark (SDU) and the Danish company LYNEX [17]. The idea was to design a modular robot having the mechanical design kept as simple as possible. The design makes it easy to build, to maintain and to modify if needed. Armadillo Scout is the first public version of the ARMADILLO, and is used at Hohenheim University as a machine platform for advanced research and teaching projects.

The Armadillo Scout field robotic tool carrier has a modular design which makes the robot configurable and adaptable to a wide range of precision agriculture research projects. The Armadillo Scout weighs about $425 \mathrm{~kg}$ and consists of two $18 \times 80 \mathrm{~cm}$ footprint belt modules with an integrated $3.5 \mathrm{~kW}$ electric motor, gear and motor controller for each belt. The belt modules are mounted on the sides of an exchangeable tool platform which allows an adjustable width and clearance height of the robot.

The Armadillo Scout is controlled by an on board computer and a Frobobox with Linux Ubuntu 11.10 as operating system. It has CAN-, Serial-, USB- and Ethernet interfaces for connecting sensors and actuators [17].

The robot is powered by a 48 VDC exchangeable Lithium Ion power pack (LFP160AH from Power Group). It is a package with 16 cells with 3.2 VDC each and connected in series. This gives a total voltage of 16 cell $\cdot 3.2 \mathrm{VDC}=$ 51.2 VDC which can be used as a 48 VDC power source and has a capacity of $160 \mathrm{Ah}$.

\section{Machine Controller Software}

The machine controller structure is divided into main machine control and implement control. While the main ma- 
Fig. 1 Autonomous tractor with GNSS based navigation and side-shift frame for mechanical weeding in row crops (inter-row hoe)

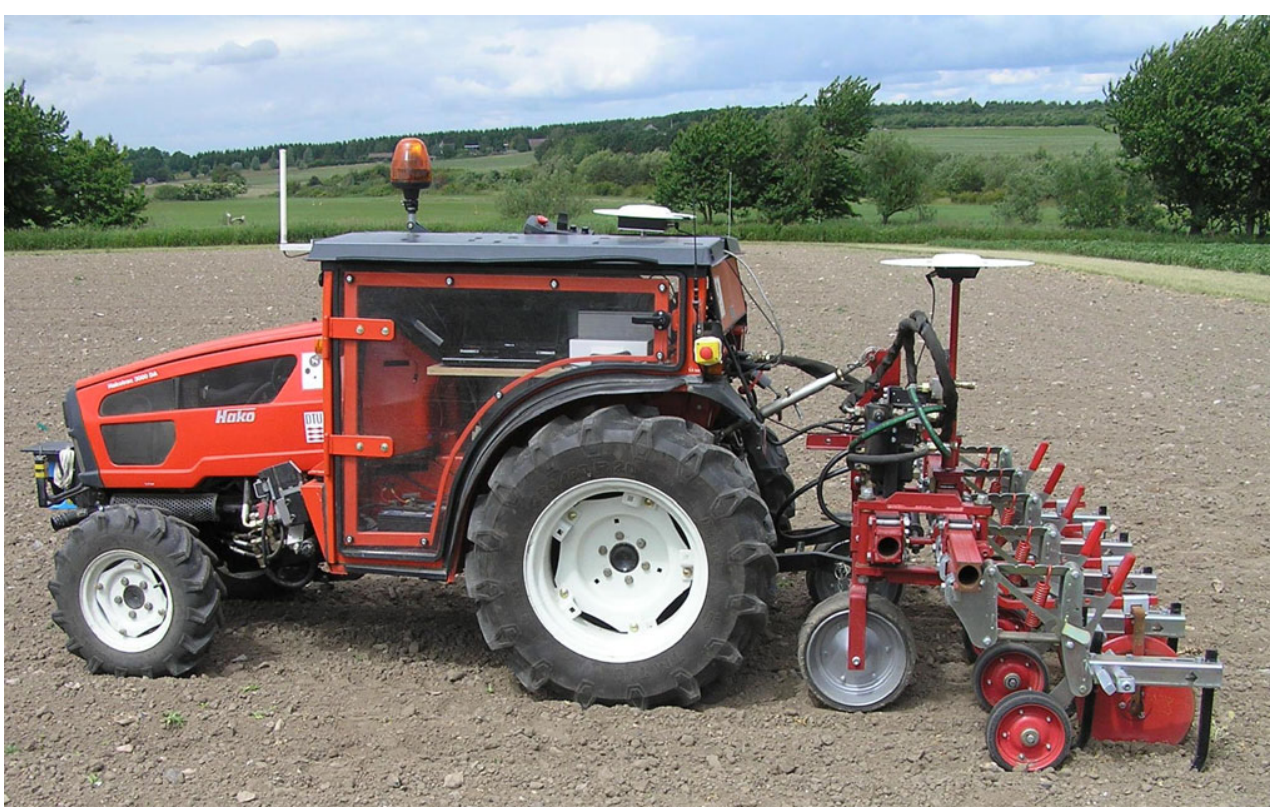

Fig. 2 Electric powered autonomous tractor with GNSS based navigation for sensing (scouting and monitoring)

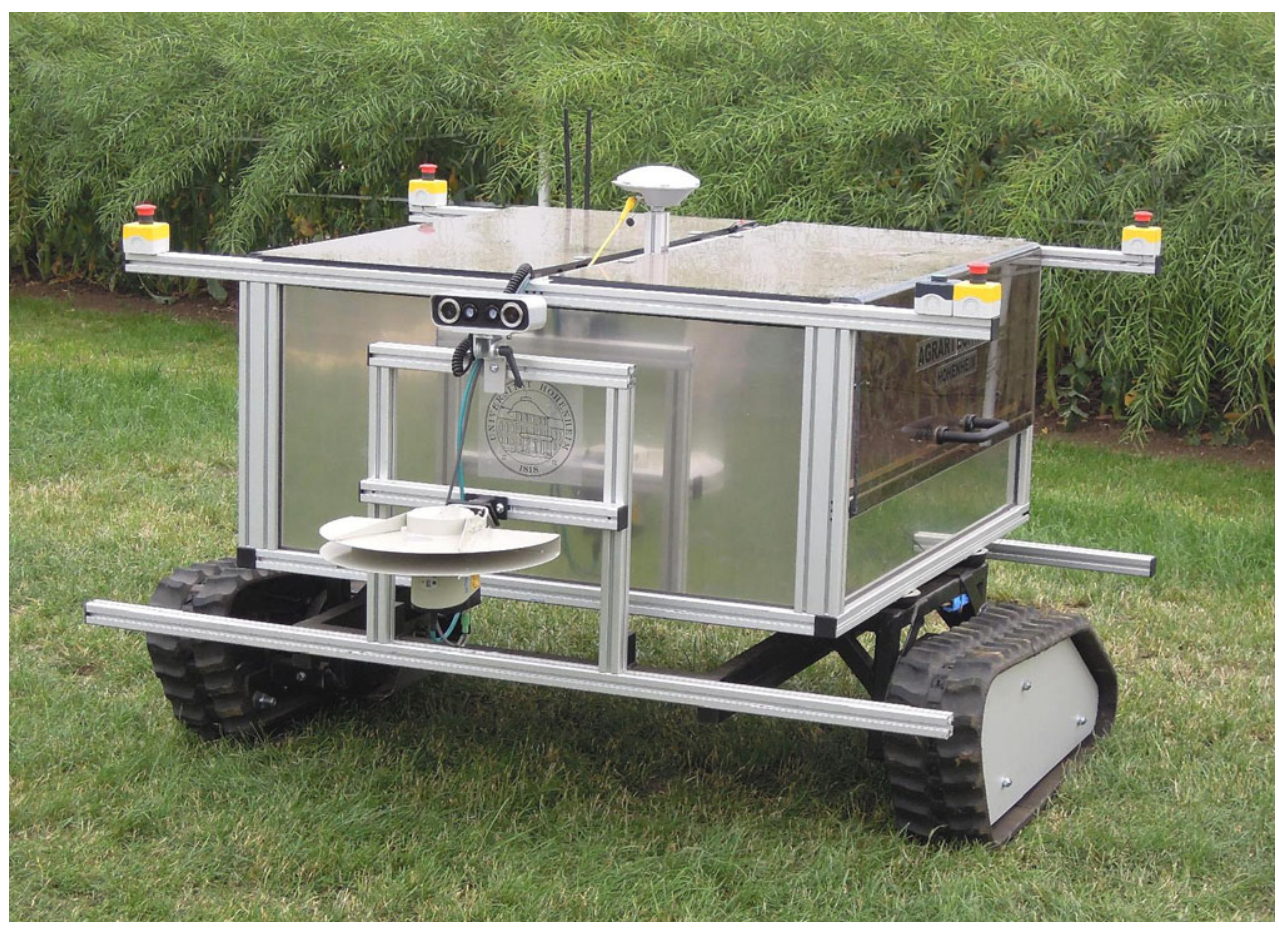

chine control is the same for the AMS and the Armadillo, the implement control software on the AMS is individual and specific because it depends on the particular application type (inter-row hoe, cycloid hoe or punch seeder) [21, 22, 25].

\subsection{Main Machine Control}

The software framework controlling the autonomous machines is called a multilayer controller due to its structure. The structure consists of a user interface including mission definition, the high and low level control and the machine interface (tractor). It is based on MobotWare developed at Denmark's Technical University (DTU). MobotWare is currently implemented on a wide range of mobile robots, from small educational indoor robots to a conventional Claas Axion 840 research prototype [1]. The Multi-Layer Controller is also implemented on both Hohenheim machines as shown in Figs. 1 and 2 [12]. The software has already been proved that it can control robots powered by combustion engines as well as by electric motors. Furthermore, it has been demonstrated that machine safety can be improved by 
Fig. 3 Overview of robot software framework MobotWare [1]

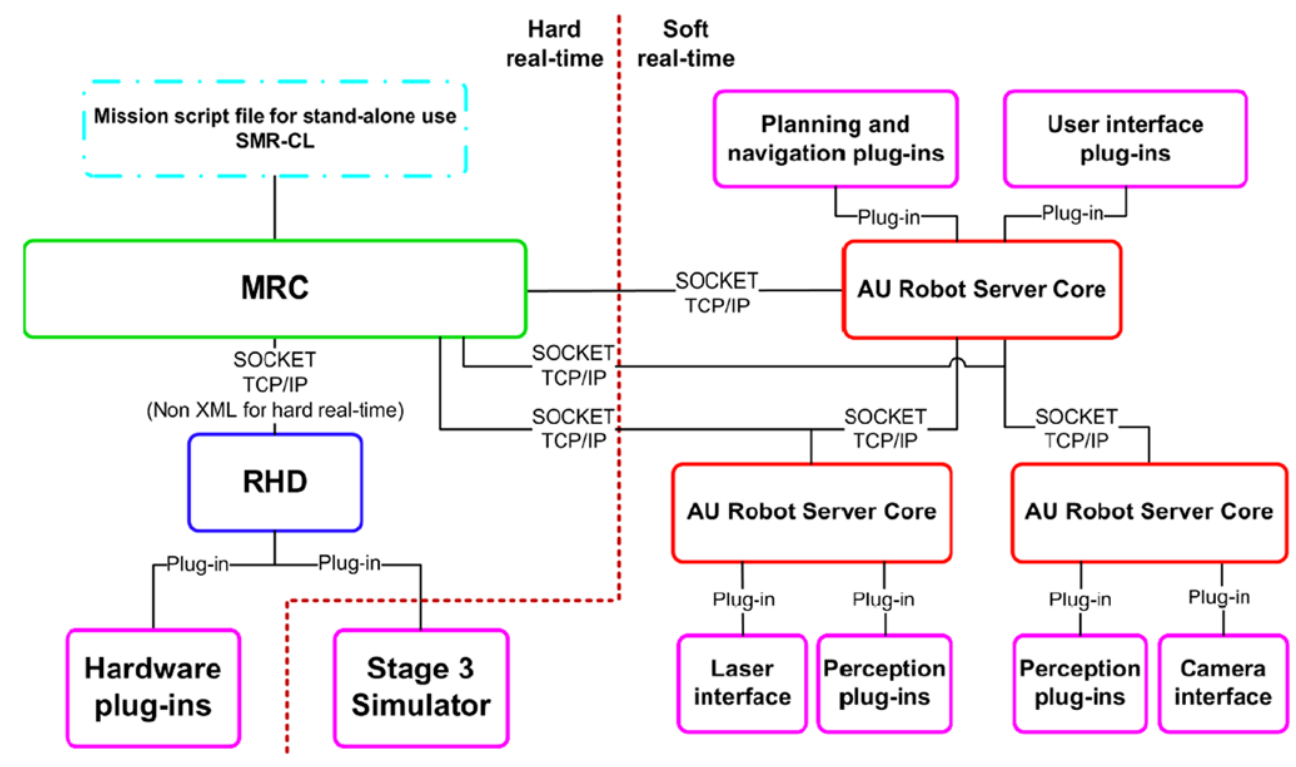

adding dedicated functionality [11]. Along with MobotWare both the AMS and the Armadillo Scout can be controlled using FroboMind, which is developed at University of Southern Denmark (SDU). FroboMind is based on ROS (Robot Operating System) and is currently being used to control the Armadillo Scout manually with a WiiMote [24].

Since development start the controller software framework has been updated and extended several times. It is described in detail in Beck et al. 2010 [1]. MobotWare is a software framework capable of controlling different mobile robot platforms operating in different environments. The framework can be divided into two sections, one with hard real-time demands and one with soft real-time demands as shown in Fig. 3. MobotWare is running under the Linux operating system. It has the option to use RTAI (Real-Time Application Interface-www.rtai.org/) to ensure real-time operation and execution of the core modules. MobotWare has three core modules [1]:

- Robot Hardware Daemon (RHD) —Flexible hardware abstraction layer for real-time critical sensors.

- Mobile Robot Controller (MRC)—Real-time closed-loop controller of robot motion and mission execution.

- Automation Robot Servers (AURS)_Advanced framework for processing of complex sensors and non-realtime mission planning and management.

\subsubsection{Robot Hardware Deamon (RHD)}

The RHD is a real-time device server, which is limited to operate only as a lightweight hard real-time hardware interface. The purpose is to handle the different requirements arising from implementations on different platforms. Based on the plug-in structure of the framework it is easy to implement new sensors or hardware to an already existing platform. The RHD also makes it easy to move the framework to a new platform, without having to reprogram the entire framework. Examples of plug-ins are Stage Simulator 2.1.1, iRobot r-Flex interface, Real Time Kinematic (RTK) and NMEA GNSS interface for Trimble and Leica Geosystems receivers, Crossbow IMU-400, PNI FieldForce ${ }^{\circledR}$ TCM, VectorNav VN-100 IMU HAKO tractor CAN-bus control interface and Claas Axion tractor CAN-bus control interface.

\subsubsection{Mobile Robot Controller (MRC)}

The MRC is a low level real-time controller for various inand outdoor mobile robots. Low level means it can control the movements and actions of the robot, but it can only execute orders. The MRC receives the orders from the high level controller or planner in order to execute a mission. The MRC is hardware independent because it uses the RHD as the hardware interface.

The MRC features are:

- Odometry,

- Motion controller,

- Small Mobile Robot-Control Language (SMR-CL) interpreter,

- Socket interface and XML-based socket interface for high level controllers or planning software,

- Socket interface to RHD,

- XML-based socket interface to sensor servers (servers running under AURS) and

- XML-based configuration.

\subsubsection{Automation Robot Servers (AURS)}

The AURS handle the non-hard real-time demands. This includes tasks like planning, mapping, localization, obstacle avoidance and image- and laser scanner analysis. The 
servers allow connections to advanced sensors like cameras and laser scanners. They are easily integrated through the plug-ins.

\subsection{Implement Control}

The implement control system software was developed in the programming and simulation tool, MatLab Simulink (MathWorks, MA, USA), to allow modeling and simulation of functionality before actual tests. MatLab also supported C-code generation, which could subsequently be executed in real-time on the implement computer. The control system updated at a rate of $40 \mathrm{~Hz}$.

The control system software for navigation of side-shift and task specific implement (inter-row hoe, cycloid hoe or punch seeder) consisted of six interdependent modules: (1) projection of global position of the GNSS antenna location onto ground level, (2) filtering and prediction in time of the global position using a Kalman filter, (3) determination of the global position of implement reference point by coordinate transformation, (4) waypoint following, (5) transverse control (side-shift), and (6) longitudinal control (tines or seeder). Once the computer was initiated, the control system software opened two specified mission files for side-shift and implement, and parsed their waypoints and additional instructions into two internal indexed copies of the files.

\subsection{Tractor and Implement Mission Planning}

Individual mission files were created before field operations for the autonomous machine and the implement (side-shift and cycloid hoe or punch seeder). Each waypoint vector contained UTM easting and northing coordinates and possible instructions. The mission files had waypoint vectors stored in successive rows and sorted in the order which defined the route which should be followed. Mission files could be edited using an ASCII text file editor. The tractor mission file contained global coordinates for vehicle route waypoints and three additional instructions, one having commands for lifting and lowering the rear three-point linkage, a second having commands for the turning procedure, and a third command for velocity. The tractor mission file was defined as a set of waypoints from and parallel to the row line. A turning command was attached to the waypoints at the ends of each row. The GNSS antennae on the tractor and the side-shift were placed at the same offset distance to the row line. An internet browser on a notebook computer that communicated with the onboard tractor computer via wireless local area network (WLAN) was used to display the graphical user interface for the navigation software. It was used for uploading the navigation waypoints to the onboard tractor computer [5, 12]. The navigation software controlled the driving parallel to row lines and the headland turns autonomously. It was supported by sensor information about GNSS-position and velocity, steering angles for the wheels and odometry status. Waypoints and their command attributes in both the tractor and side-shift mission files were planned in such a way that, when the tractor lowered the three-point linkage, the side-shift and implement would be activated, as indicated by the joint instruction. When the tractor lifted the three-point linkage, the side-shift and implement would be deactivated. More details are described in the specific publications [21, 22, 25].

\section{Field Operations and Applications}

New crop management strategies within Precision Farming divide fields up into sub-fields of homogeneous soil or crop properties to address spatial variability by varying input dose rates per sub-field. Research has been conducted with higher spatial resolutions for targeting individual plants [27]. These advanced systems aim at sensing individual plant status and stresses and at targeting mechanical treatments and inputs in small dose rates adapted to individual crop plant needs (Plant Scale Husbandry) [7, 8].

\subsection{Crop Establishment}

For the crop establishment in combination with the AMS a passive data logging system for seed mapping, an active grid seeder and a punch planter were developed [9, 25]. The general aim was to accurately place seeds at known positions and thereby to ease individual crop plant treatments as e.g. for automated mechanical weeding operations.

For seed mapping a conventional passive seeder was equipped with a data logger and an RTK GNSS receiver. During the seeding operation when seeds dropped into the furrow, time and position data were recorded. In a post processing procedure the data were analyzed and each seed could be geo-referenced. For subsequent field operations these absolute seed positions can be used e.g. for steering purposes because seed position coordinates indicate also the location of rows. Row positions allow weeding parallel to or between crop rows and single seed positions allow even individual crop plant treatments within rows [9].

To arrange plants in regular grids, a special actively controlled seeder was developed which allows unit drive synchronisation within the working width, a synchronisation between passes and a very accurate control of the lateral row spacing between successive passes (side-shift) [10]. For achieving highly even plant grids a very accurate seed spacing is required.

In general seed placement is optimised in two dimensions in longitudinal and transversal directions. Results from the machine testing in the lab showed that the motors were 
Fig. 4 Autonomous tractor with GNSS based navigation and side-shift frame for highly accurate placement of seeds (punch planter)

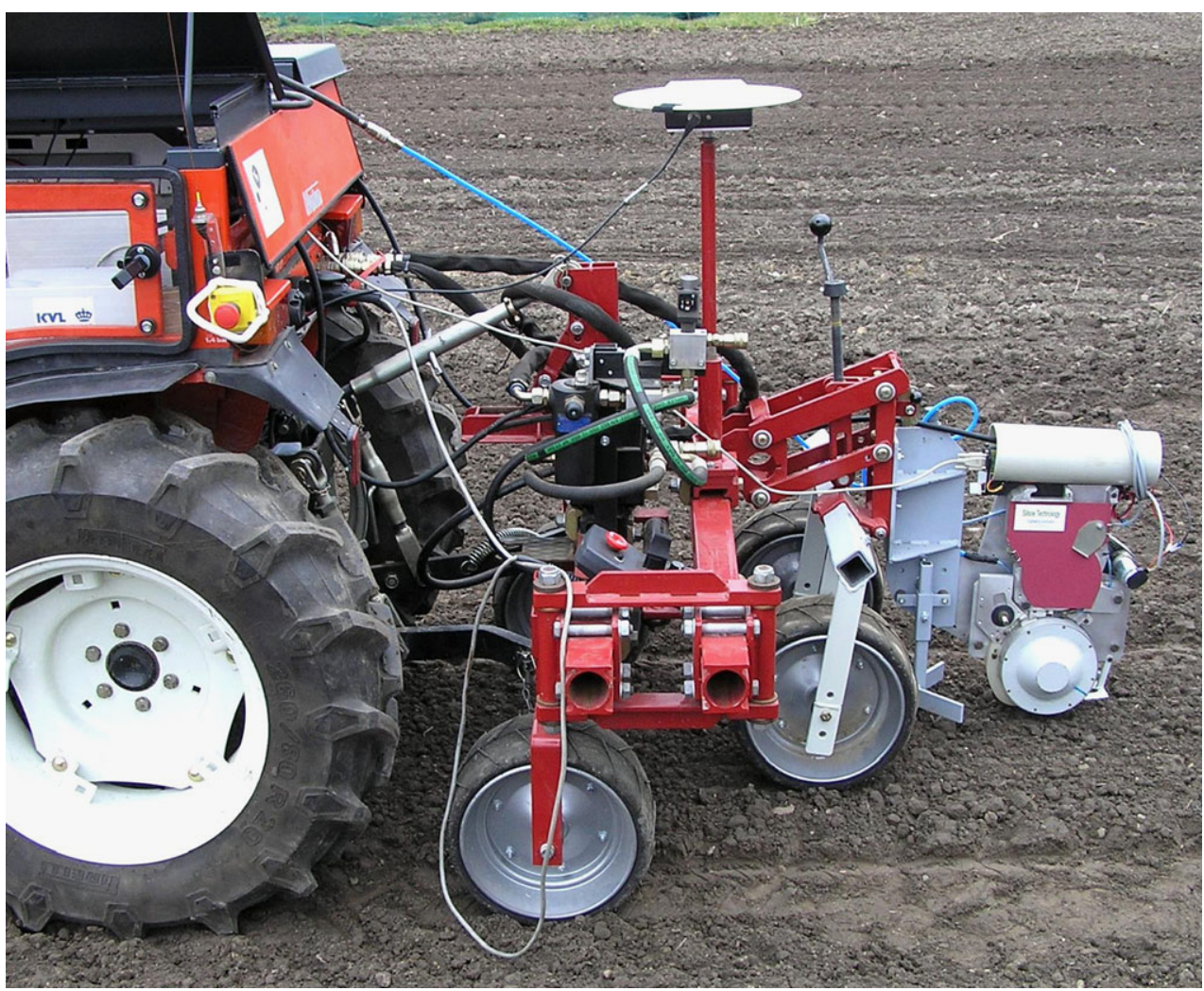

able to drive the discs at a constant and very accurate speed. The standard deviations for the accumulated time per seed detection for the same spacing sequence between the four seeders were calculated. This parameter allows the evaluation of the synchronization between the rows. The mean of standard deviations was $4.23 \mathrm{~ms}$. The test run was conducted with a $0.597 \mathrm{~m} / \mathrm{s}$ forward speed. The mean absolute standard deviation was calculated to $2.53 \mathrm{~mm}$. Assuming a normal distribution, $95 \%$ of the data were in a range of $\pm 5.1 \mathrm{~mm}$. Grid seeding with such a spacing accuracy has to be regarded as sufficient and very accurate. However, field experiments have to be conducted to investigate the effects under dynamic operation and field conditions [9].

Punch planting was developed for the autonomous machine based on a modified one-row lightweight dibber drill [18] (Fig. 4). The mechanical drive system was substituted by an electric motor directly attached to the wheel housing. A microcontroller and a RTK GNSS were used to control the speed of the seeder wheel. Seeds were pressed into the soil as a dibber was extended pneumatically out of the seeder wheel leaving open holes. Finally, the dibber rapidly retracted leaving a neat hole in which the seed is left firmly embedded in its base. The seeds were spaced $5 \mathrm{~cm}$ apart within the row. The circumferential speed of the seeding aggregate (wheel) was kept the same as the forward speed by the electronic controller. This created well shaped holes and avoided (i) the wheel sliding over the field surface and (ii) the dibbers disturbing more soil than necessary [25].

\subsection{Crop Management}

Reducing herbicide use is an aim of crop management. Accurate inter-row hoeing requires accurate lateral control of tools and is mainly provided by a second operator. For this purpose row detection sensors help to automate the tool control as well as the tractor navigation [26]. But these field operations are still challenging due to its sometimes low weeding efficacy or high crop plant losses and high labor intensity. An automatic inter-row hoe as well as an intra-row rotor weeder was developed. It was possible to mount them on the autonomous platform. Vehicle navigation between rows for implements and tractors as well as the guidance of treatment tools can be based on seed map information.

For inter-row hoeing a conventional hoe was equipped with an electro-hydraulic side-shift frame and a RTK-GNSS based controller (Fig. 1) [10]. The GNSS antenna was placed on the implement in order to be closer to the location of interest (tool positions). The main task of the hoe controller system was to minimize the lateral deviations between actual positions of the hoe related to a predefined route. The hoe system achieved an increase of the accurate guidance and enabled hoeing up to $83 \%$ of a field surface area. The GNSS based system showed its potential to be used for high accurate crop row guidance without a second operator [10].

Weeding within crop rows has not yet been automated to a satisfactory level. It is today often still performed by 
humans or a herbicide sprayer treating only the row area (strip application). A controller system for a weeding rotor (cycloid hoe) with individual tine switching was developed to allow tillage within crop rows [22]. Main parameters to achieve a particular tillage effect are the ratio of forward speed to rotational speed, the diameter of tine rotation, the lateral offset to crop rows, the number of tines and the shape or design of tine tips. The system consisted of a tine rotor including a parallelogram based attachment, ground wheel for depth control, hydraulic motor, speed deducting gearbox and a GNSS based controller. The tine rotor has eight tines that are placed in a circle around an axis. The combination of the circular movement of the tines and the linear forward movement of the tractor leads to a cycloidal path for the tines. Each tine is mounted on an individual electrically activated spindle that enables two switching modes. In the locked mode, the tines move into the row, and in the un-locked mode, they keep outside the row to avoid collision with the crop plants. A control of the rotor is needed to avoid crop plant damages. By knowing both the individual crop plant positions (seed map and/or optical sensor) and the current rotor position the absolute distance between tine circumference and crop plant can be calculated. Mechanical clutches within the rotor are activated as the crop distance falls below a definable value (trigger distance). By assuming that conventional inter-row hoeing covers up to around $80 \%$ of a total field surface additional intra-row weeding by a rotor weeder increases the result to up to almost $90 \%$ [22].

\section{System Safety and Reliability}

A mobile robot operating in semi-natural environments like fields, orchards or plantations must cope with a high degree of structural diversity. Furthermore, internal machine faults can result in unwanted machine behaviors and can even result in an unsafe machine performance status. Therefore, the machine must be 'aware' of its close environment to carry out operations efficiently and also to avoid collisions with other objects that could damage the machine and the other objects (infrastructure, plants, humans and animals). To meet these performance goals automated perception capabilities are required, and the robot must react promptly and appropriately when unexpected objects are detected or when faults of various severity occurs. A main focus of outdoor robotic research is the development of agent-based architectures suitable for unmanned, possibly unattended, but still supervised systems [6].

High levels of reliability and safety are necessary and can be achieved by novel developments within automated perception, diagnosis and decision making and fault tolerant operation. To achieve these aims extra functionality has to be added so that the machine will behave in a safe, reliable and effective manner under unmanned operation [11]. The machine system and the operation conditions were assessed by a machine safety consultant to check the compliance of existing legal requirements. Based on legal safety consultancy and a failure modes and effects analysis a redesign of the machine system was completed [11]. The machine will be operating unmanned but not unattended. An operator prepares, checks and starts the machine in the operation area. During machine operation the operator is around and is supposed to complete other working tasks. An additional stereo vision camera and a laser scanner will provide more information about the machine environment to avoid collision with obstacles. They also improve the machine navigation and will contribute to reduce the dependency on GNSS information. Signal monitoring software was developed to achieve higher levels of reliable and fault tolerant control. An additional safety circuit including bumper switches allow the machine to stop when other collision sensors fail.

The hardware result from the research project is the redundant safety circuit. The circuit is compliant with the DS/EN ISO 13849-1 standard for machine safety. The safety circuit cuts off the fuel injection to the engine if one of the emergency buttons is pressed or the RFID switches of the front bumper are activated. Additionally a wireless emergency switch controlled by the machine operator can stop the machine within a range of $100 \mathrm{~m}$ during the operation. When the tractor is not running in autonomous mode it can be guided by a wired remote control. The operator is using it to move the machine for transportation purposes.

\section{Conclusions}

A multilayer controller has successfully been implemented on two outdoor machines to conduct several agricultural applications in autonomous mode. The University of Hohenheim has successfully collaborated with national and international research partners and commercial companies in developing autonomous machines with the necessary perception and controller frameworks. Future work has to be conducted to achieve a more integrated and flexible implement control. A strategy could be to allow an implement control based on the ISO 11783 standard. This would even allow or at least ease the integration of existing conventional implements based on this ISO standard.

Acknowledgements We gratefully acknowledge the support of our partners S. Christensen, H. Nielsen, J. Nielsen, J. Rasmussen from University of Copenhagen, Denmark; O. Ravn, N.A. Andersen, M. Blanke, J.C. Andersen from Denmark's Technical University; K. Jensen from University of Southern Denmark; M. Nørremark, R.N. Jørgensen from Aarhus University, Denmark; B.S. Blackmore from Harper Adams University College, UK; S. Fountas from University of Thessaly, Greece; O. Heinemann from Hako Werke GmbH, Germany. 


\section{References}

1. Beck AB, Andersen NA, Andersen JC, Ravn O (2010) MobotWare-a plug-in based framework for mobile robots. In: Proceedings IFAC symposium on intelligent autonomous vehicles, July 2010, Lecce, Italy, International Federation of Automatic Control (IFAC)

2. Berns K, Kuhnert KD, Armbrust C (2011) Off-road robotics-an overview. Künstl Intell 25:109-116

3. Blackmore BS, Griepentrog HW (2006) Autonomous vehicles and robots. In: Munack A (ed) CIGR handbook of agricultural engineering. Information technology, vol VI. ASABE, St Joseph, pp 204-215. Chap. 4.3

4. Blackmore BS (2007) A systems view of agricultural robots. In: Stafford JV (ed) Proceedings 6th European conference on precision agriculture (ECPA), June 2007, Skiathos, Greece. Wageningen Press, Wageningen, pp 23-31

5. Bochtis DD, Vougioukas S, Griepentrog HW (2009) A mission planner for an autonomous tractor. Transact ASABE 52(5):14291440

6. Granot R (2001) Telerobotics a new paradigm. In: International symposium on mechatronics: the challenges of the new millennium in research and education. Keynote, November 9, 2001, Budapest

7. Griepentrog HW, Norremark M, Nielsen H, Blackmore BS (2003) Individual plant care in cropping systems. In: Stafford JV, Werner A (eds) Proceedings 4th European conference on precision agriculture (ECPA), 26 June 2003, Berlin. Wageningen Academic Press, Wageningen, pp 247-251

8. Griepentrog HW, Norremark M, Nielsen H, Blackmore BS (2005) Seed mapping of sugar beet. Precis Agric 6(2):157-165

9. Griepentrog HW, Skou PT, Soriano JF (2005) Design of a seeder to achieve highly uniform sowing patterns. In: Stafford JV, Thylen L (eds) Proceedings 5th European conference on precision agriculture (ECPA), 2005, Uppsala, Sweden, pp 675-682

10. Griepentrog HW, Nørremark M, Nielsen J, Soriano Ibarra J (2007) Autonomous inter-row hoeing using GPS based side-shift control. In: Agricultural engineering international-the CIGR ejournal IX (Manuscript ATOE 07 005)

11. Griepentrog HW, Andersen NA, Andersen JC, Blanke M, Heinemann O, Nielsen J, Pedersen SM, Madsen TE, Wulfsohn D (2009) Safe and reliable-further development of a field robot. In: van Henten EJ, Goense D, Lokhorst C (eds) Proceedings 7th European conference on precision agriculture (ECPA), 6 July 2009. Wageningen Academic Publishers, Wageningen, pp 857-866

12. Griepentrog HW, Jaeger-Hansen CL, Ravn O, Andersen NA, Andersen JC, Nakanishi T (2011) Multilayer controller for field robots-high portability and modularity to ease implementation. Paper presented at LAND.TECHNIK-AgEng 2011, Hanover, Germany

13. Griepentrog HW, Jaeger-Hansen CL, Dühring K (2012) Electric agricultural robot with multi-layer-control. In: Proceedings CIGRAgEng, 8 July 2012, Valencia, Spain

14. Grift T, Zhang Q, Kondo N, Ting KC (2008) A review of automation and robotics for the bioindustry. J Biomech Eng 1(1):37-54

15. Hollingum J (1999) Robots in agriculture. Ind Robot 26(6):438446

16. Im KY, Oh SY, Han SJ (2002) Evolving a modular neural networkbased behavioural fusion using extended VFF and environment classification for mobile robot navigation. IEEE Trans Evol Comput 6(4):413-419

17. Jensen K, Nielsen SH, Joergensen RN, Boegild A, Jacobsen NJ, Joergensen OJ, Jaeger-Hansen CL (2012) A low cost, modular robotics tool carrier for precision agriculture research. In: Proceedings 11th international conference on precision agriculture, Indianapolis, USA

18. Miles SJ, Reed JN (1999) Dibber drill for precise placement of seed and granular pesticide. J Agric Eng Res 74(2):127-133

19. Motlagh O, Tang SH, Ismail N, Ramli R (2012) An expert fuzzy cognitive map for reactive navigation of mobile robots. Fuzzy Sets Syst 201:105-121

20. Na YK, Oh SY (2003) Hybrid control for autonomous mobile robot navigation using neural network based bahaviour modules and environment classification. Auton Robots 15:193-206

21. Nørremark M, Griepentrog HW, Nielsen J, Søgaard HT (2008) The development and assessment of the accuracy of an autonomous GPS-based system for intra-row mechanical weed control in row crops. Biosyst Eng 101(4):396-410

22. Nørremark M, Griepentrog HW, Nielsen J, Søgaard HT (2012) Evaluation of an autonomous GPS-based system for intra-row weed control by assessing the tilled area. Precis Agric 13(2):149162

23. Pedersen SM, Fountas S, Have H, Blackmore BS (2006) Agricultural robots - system analysis and economic feasibility. Precis Agric 7(4):295-308

24. Quigley M, Gerkey B, Conley K, Faust J, Foote TB, Leibs J, Berger E, Wheeler R, Ng A (2009) ROS—an open-source robot operating system. In: Proceedings ICRA workshop on open source software, 12-17 May 2009, Kobe, Japan

25. Rasmussen J, Griepentrog HW, Nielsen J, Henriksen CB (2012) Automated intelligent rotor tine cultivation and punch planting to improve the selectivity of mechanical intra-row weed control. Weed Res 52(4):327-337

26. Tillett ND (1991) Automatic guidance sensors for agricultural field machines—a review. J Agric Eng Res 50(3):167-187

27. Tillett ND, Hague T, Marchant JA (1998) A robotic system for plant-scale husbandry. J Agric Eng Res 69:169-178

28. van Straten G (2004) Field robot event, Wageningen, 5-6 June 2003. Computers and electronics in agriculture, vol 42, pp 51-58

29. Yavuz H, Bradshaw A (2002) A new conceptual approach to the design of hybrid control architecture for autonomous mobile robots. J Intell Robot Syst 34:1-26

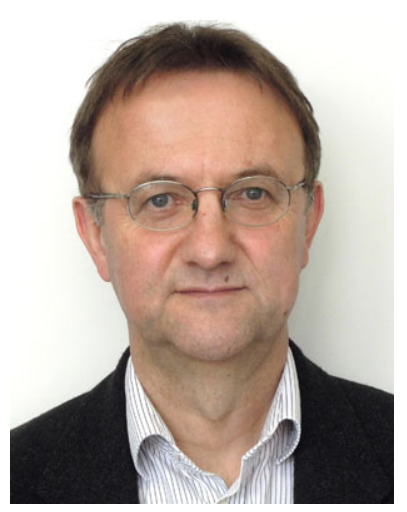

H.W. Griepentrog holds a M.Sc. in mechanical engineering from the Technical University in Berlin, Germany and received a Ph.D. in agricultural sciences from Kiel University, Germany. Since 1997 he was Associate Professor for automation \& precision farming at the University of Copenhagen, Denmark and since 2011 he is a full professor at the University of Hohenheim, Germany. Present research activities are in the area of instrumentation \& test engineering and robotics \& precision farming. 


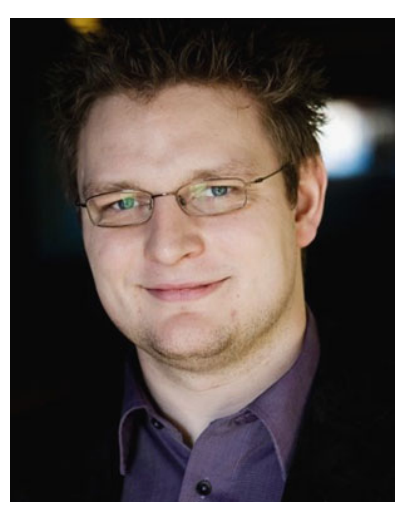

C.L. Dühring Jaeger received his M.Sc.E. from Technical University of Denmark in 2010. Since 2011 he has been at University of Hohenheim, Germany as a research assistant and since 2013 he is a Ph.D. candidate. His research interests include behavior based control systems, autonomous mobile robots, and robot navigation.

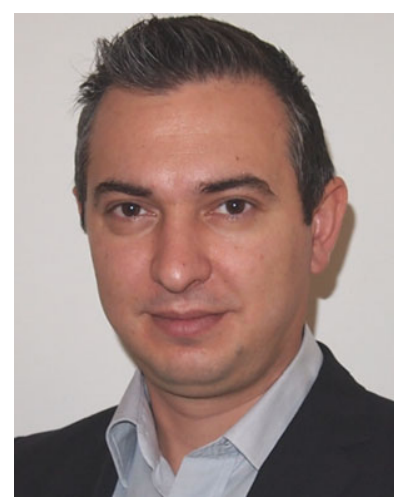

D.S. Paraforos received his B.Sc. in automation engineering from Chalkis Institute of Technology, Greece and his M.Sc. in agricultural engineering from University of Thessaly, Greece in 2005 and 2008, respectively. Since 2012 he has been at University of Hohenheim, Germany as a research assistant and since 2013 he is a Ph.D. candidate. His research interests include robotics and automation and fatigue life of agricultural machinery. 